\title{
Inteligencia emocional, género y clima familiar en adolescentes peruanos
}

\author{
Percy Ruiz ${ }^{1 * 2}$, , Renzo Felipe Carranza Estebann ${ }^{2,3}$ \\ ${ }^{1}$ Universidad San Ignacio de Loyola, Lima, Perú; ${ }^{2}$ Universidad Peruana Unión, Lima, Perú; ${ }^{3}$ Universidad Peruana Unión, Tarapoto, Perú.
}

Recibido, febrero 15/2017

Concepto de evaluación, abril 10/2017

Aceptado, febrero 19/2018
Referencia: Ruiz, P., \& Carranza, R.F. (2018). Inteligencia emocional, género y clima familiar en adolescentes peruanos. Acta Colombiana de Psicología, 21(2), 188-199. doi: http://www.dx.doi.org/10.14718/ ACP.2018.21.2.9

\section{Resumen}

\begin{abstract}
El objetivo del presente estudio fue analizar la relación entre la inteligencia emocional y el clima familiar. Se realizó un estudio transversal-correlacional en el que participaron 127 adolescentes del distrito de San Juan de Lurigancho, en Lima, Perú, donde se obtuvo información sobre su edad, género y estructura familiar. Las mediciones del clima familiar (CF) e inteligencia emocional (IE) se hicieron a través de una versión adaptada de la escala del clima social familiar (FES) y una escala de inteligencia emocional. Se utilizó la prueba estadística t de Student para la comparación de las puntuaciones de inteligencia emocional según el género y la estructura familiar, y la $\mathrm{r}$ de Pearson para el análisis de correlaciones entre el clima familiar y la inteligencia emocional. Como resultado, se encontraron diferencias de género en empatía $(\mathrm{t}=3.445 ; \mathrm{p}<.01)$ y habilidades sociales $(\mathrm{t}=2.711 ; \mathrm{p}<.01)$-donde las mujeres presentaron puntuaciones más altas que los hombres-, pero no en la puntuación total de la inteligencia emocional. También, se encontraron diferencias significativas en la autorregulación $(\mathrm{t}=2.319 ; \mathrm{p}<.05)$ y automotivación $(\mathrm{t}=2.713 ; \mathrm{p}<.01)$ de los adolescentes de familias nucleares y monoparentales; y se observó una correlación directa entre el clima familiar y la inteligencia emocional $(r=.632 ; \mathrm{p}<.01)$.

Palabras clave: familia, estructura familiar, clima familiar, inteligencia emocional, adolescentes.
\end{abstract}

\section{Emotional intelligence, gender and family environment in Peruvian adolescents}

\begin{abstract}
The objective of this study was to analyze the relationship of emotional intelligence and family environment. A cross-sectional and correlational study was executed, involved 127 adolescents in San Juan de Lurigancho District, Lima - Peru. Data obtained were on age, sex, and family structure. Measurements of family environment (FE) and emotional intelligence (EI) were made through an adapted version of the Family Environment Scale (FES) and a EI scale, respectively. Student's t test was used for the comparison of emotional intelligence scores by gender and family structure and Pearson's $r$ for the correlation analysis between family environment and emotional intelligence. The analysis revealed gender differences in empathy $(\mathrm{t}=3.445$, $\mathrm{p}<.01)$ and social skills $(\mathrm{t}=2.711, \mathrm{p}<.01)$, where women presented higher scores than men. There were no gender differences in the total score of EI. There were also significant differences in self-regulation $(t=2.319 ; \mathrm{p}<.05)$ and self-motivation $(\mathrm{t}=2.713 ; \mathrm{p}<.01)$ in adolescents from nuclear and non-nuclear families. Finally, a direct correlation between family environment and emotional intelligence was found $(\mathrm{r}=.632, \mathrm{p}<.01)$.

Key words: gender, family, family structure, family environment, emotional intelligence, adolescents.
\end{abstract}

* Centro de Investigación. Urb. La Alameda de Naña, A-7, Lurigancho, Lima, Perú.+51 01946854448. percygruiz@hotmail.com 


\title{
Inteligência emocional, gênero e clima familiar em adolescentes peruanos
}

\author{
Resumo
}

\begin{abstract}
O objetivo do presente estudo foi analisar a relação entre a inteligência emocional e o clima familiar. Realizou-se um estudo transversal-correlacional no qual participaram 127 adolescentes do distrito de San Juan de Lurigancho, em Lina, no Peru, onde obteve-se informação sobre sua idade, gênero e estrutura familiar. As medições do clima familiar (CF) e inteligência emocional (IE) foram feitas através de uma versão adaptada da escala do clima social familiar (FES) e uma escala de inteligência emocional. Utilizou-se o teste estatístico t de Student para a comparação das pontuações de inteligência emocional segundo o gênero e a estrutura familiar, e a $r$ de Pearson para a análise de correlações entre o clima familiar e a inteligência emocional. Como resultado, foram encontradas diferenças de gênero em empatia $(t=3.445 ; \mathrm{p}<.01)$ e habilidades sociais $(\mathrm{t}=2.711$; $\mathrm{p}<.01$ ), onde as mulheres apresentaram pontuações mais altas do que os homens, mas não na pontuação total da inteligência emocional. Também, foram encontradas diferenças significativas na autorregulação $(\mathrm{t}=2.319 ; \mathrm{p}<.05)$ e automotivação $(\mathrm{t}=2.713 ; \mathrm{p}<.01)$ dos adolescentes de famílias nucleares e monoparentais e observou-se uma correlação direta entre o clima familiar e a inteligência emocional $(\mathrm{r}=.632 ; \mathrm{p}<.01)$.

Palavras-chave: adolescentes, clima familiar, estrutura familiar, família, inteligência emocional.
\end{abstract}

\section{INTRODUCCIÓN}

Las primeras investigaciones sobre la inteligencia emocional (IE) consistieron en examinar dicho constructo por medio del desarrollo de modelos teóricos y la creación de instrumentos de evaluación (Mayer, Caruso \& Salovey, 1999). En la actualidad, este tema sigue recibiendo una considerable atención por parte de investigadores en los campos de la psicología (AlDosiry, Alkhadher, AlAqraa' \& Anderson, 2015), y, recientemente, se han realizado investigaciones empíricas enfocadas en la constatación de los efectos que puede tener la inteligencia emocional sobre los individuos (Sánchez, León \& Barragán, 2015) y en el desarrollo de habilidades emocionales (Herpertz, Schütz y Nezlek, 2016).

Según Mayer y Salovey (1997), la inteligencia emocional se define como "la capacidad para percibir, asimilar, comprender y regular las emociones propias y de los demás, promoviendo un crecimiento emocional e intelectual" (p. 10); y se sabe que, a diferencia del coeficiente intelectual -considerado como relativamente estable-, las habilidades de la inteligencia emocional pueden mejorarse a través del aprendizaje y la práctica (Goleman, 2011), y su desarrollo implicaría el éxito de los individuos en diversos campos de actuación.

Autores como Bar-On (1997), Gottman, (1997), Cooper y Sawaf (1998), Shapiro (2010) y Goleman (2011) han propuesto diversas aproximaciones al concepto de inteligencia emocional y han elaborado instrumentos de medición, cada uno con sus propios componentes. Sin embargo, aunque existe una discrepancia entre los conceptos y las habilidades emocionales que debe poseer un individuo emocionalmente inteligente, también existe un consenso con respecto a que estas tienen una influencia sobre diferentes aspectos de la vida (Extremera \& Fernández, 2004); aspectos que están siendo contrastados de forma empírica en diversos estudios recientes.

Al respecto, la revisión de literatura muestra investigaciones en las que se estudió la relación de la inteligencia emocional con variables como la edad, el género y la cultura (McNulty, Mackay, Lewis, Lane \& White, 2015), el apoyo social (Azpiazu, Esnaola y Sarasa, 2015), el rendimiento de ventas (AlDosiry et al., 2015), la calidad de vida y la consecución de objetivos laborales (Macías, Gutiérrez, Carmona \& Crespillo, 2015), y la autoeficacia y las intenciones emprendedoras (Mortan, Ripoll, Carvalho \& Bernal, 2014). Donde, en la mayoría de las investigaciones desarrolladas en entornos educativos, se tuvo como objetivo el análisis de relaciones entre la inteligencia emocional y constructos como el rendimiento académico (Aritzeta et al., 2016), la inteligencia general y el bienestar psicológico (Sánchez et al., 2015), y la conciencia emocional y la inadaptación (Ordóñez, Maganto \& González, 2015).

\section{Inteligencia emocional}

Desde de la perspectiva teórica de la evaluación cognitiva, las emociones son el resultado de los estímulos personales más intensos, que se caracterizan por ser relativamente de corta duración, tener un objetivo específico e implicar la activación fisiológica y las tendencias de comportamiento (Bagozzi, Gopinath \& Nyer, 1999; Johnson \& Stewart, 2005).

Asimismo, autores como Fernández y Extremera (2013) sostienen que la inteligencia académica no es suficiente para alcanzar el éxito profesional, así como que tampoco 
garantiza el éxito en la vida cotidiana y que no contribuye en el equilibrio emocional y la salud mental; mientras que la inteligencia emocional sí lo hace, ya que incluye emociones internas que son importantes para el crecimiento personal y el ajuste emocional.

Inicialmente, el concepto de inteligencia emocional cobró importancia por las consecuencias sobre la crianza y educación de los niños, pero, posteriormente, su investigación se extendió a los entornos laborales, sobre todo en torno a las relaciones humanas, donde se asume que las mismas capacidades del control emocional que hacen que un niño sea apreciado y considerado como entusiasta por los demás también le ayudarán cuando interactúe en el trabajo y el matrimonio (Shapiro \& Tiscornia, 1997).

Específicamente, según la teoría de las inteligencias múltiples de Gardner (2006), la inteligencia intrapersonal es presentada como un conjunto de habilidades de comprensión, control y regulación de las propias emociones, mientras que la inteligencia interpersonal es definida como las habilidades de comprensión de las emociones de los demás. Y, teniendo esto en cuenta, Goleman (2011) propone que la inteligencia emocional es un conjunto de habilidades emocionales que pueden desarrollarse y que se caracterizan por contar con cinco componentes: el autoconocimiento, la autorregulación, la automotivación, la empatía y las habilidades sociales. Sin embargo, según Fernández y Extremera (2013), aunque todas estas habilidades forman parte de la inteligencia emocional, los aspectos personales e interpersonales son bastante independientes y no necesariamente tienen que presentarse en forma concadenada.

En este sentido, algunos estudios han mostrado que el autoconocimiento está relacionado con altos índices de comportamiento basado en competencias y con menores puntuaciones en escalas de dependencia y conducta agresiva (Aimaganbetova et al., 2016); mientras que la autorregulación se ha asociado negativamente con problemas emocionales y de comportamiento en los niños (Eisenberg, Spinrad \& Eggum, 2010); el autocontrol puede ser imposibilitado por las emociones negativas (Chester et al., 2016); y la empatía se ha asociado negativamente a un comportamiento de intimidación (Mitsopoulou \& Giovazolias, 2015).

\section{Género e inteligencia emocional}

Hace décadas que los investigadores han puesto énfasis en el estudio del género desde una perspectiva biológica; sin embargo, en los últimos veinticinco años se han realizado enormes esfuerzos para lograr que la comprensión del género sea vista como un fenómeno cultural (Conway, Bourque \& Scott, 2013).
Antiguamente, Parsons y Bales (1956), por ejemplo, creían que los roles de género tienen un fundamento biológico, que los procesos de modernización de las estructuras sociales habían logrado justificar el establecimiento de estos roles, y que los sistemas sociales solo son funcionales si tienen como base la complementariedad y el apoyo mutuo entre hombres y mujeres; lo que conllevaría a que las variaciones en las funciones correspondientes al género fueran vistas como desviaciones que traen como consecuencia la desaparición de dichos sistemas sociales.

Sin embargo, en respuesta a la concepción biológica del género, posteriormente resurgieron las propuestas teóricas de Med (1935), que han servido como base para la comprensión del género basado en aspectos culturales y en un mayor entendimiento de la compleja funcionalidad de los sistemas sociales; lo que quiere decir que los roles de género presentan una variación histórica y cultural, y son entendidos como representaciones culturales establecidas por la sociedad sobre el comportamiento apropiado para hombres y mujeres, y en el que la interacción de diversas instituciones económicas, sociales, políticas y religiosas cumplen una función mediadora (Conway et al., 2013). Actualmente, la necesidad del estudio del género está vigente y es de gran importancia debido a que el rol de las mujeres en la sociedad no solo es un tema presente en los debates políticos, sino que tiene implicaciones sociales y económicas que, por tanto, suscitan problemas intelectuales entre los académicos e intelectuales.

Teniendo en cuenta lo anterior, queda claro que la comprensión del género y sus implicaciones en otros aspectos de la sociedad pueden ser abordadas desde dos perspectivas que, más que excluyentes, pueden considerarse complementarias, ya que una concepción biológica del género delimita su estudio a elementos concretos y reduccionistas, mientras que una concepción cultural exige la inclusión de elementos propios del contexto, lo que hace que el estudio de dichos roles sea algo mucho más complejo.

Aun cuando no hay certeza sobre el origen de las similitudes y diferencias de género en cuanto a las emociones y conductas (Eagly, Beall \& Sternberg, 2004), sí existen estudios que han demostrado las diferencias de género en aspectos como la empatía y otros conceptos. Al respecto, Riglin et al. (2016), por ejemplo, señalan que las diferencias de género en cuanto a la inteligencia no están claramente demostradas, pero argumentan que hay indicios de que la salud mental de las mujeres se ve menos afectada que la de los hombres; por su parte, Egger y Angold (2006) afirman que las niñas tienen menos riesgo de problemas de conducta, con respecto a los niños; y otros autores sostienen que las mujeres son emocionalmente más expresivas que los 
hombres, que son más empáticas y perceptivas -debido a que su comprensión y reconocimiento de las emociones de los demás es mayor-y que presentan mayor habilidad en ciertas competencias interpersonales (Argyle, 1990; Lafferty, 2004). Sin embargo, existe controversia con respecto a las diferencias de género en la inteligencia emocional, pues dependiendo del tipo de instrumento de evaluación se pueden o no descubrir dichas diferencias (Sánchez, Fernández, Montañés \& Latorre, 2008).

Clima familiar, estructura familiar e inteligencia emocional

La familia es considerada como la unidad básica de la sociedad y, como tal, es el agente fundamental e imprescindible de socialización (Lekaviciene \& Antiniene, 2016; Povedano, Hendry, Ramos \& Varela, 2011). Como objeto de estudio, en las últimas décadas la familia ha recobrado el creciente interés de los investigadores, sobre todo debido a los cambios sustantivos y progresivos del comportamiento familiar, los cuales abarcan múltiples procesos -desde la constitución familiar hasta sus distintas formas de vida(Paredes, 2003), ya que existen actualmente diversos modelos familiares que difieren de los parámetros de entendimiento de la vida familiar (Valdivia, 2008) y que, al parecer, no existe una norma estándar ni tampoco un modelo de familia contemporánea (Jadue, 2003).

Al respecto, la revisión teórica sugiere una relación entre algunos aspectos familiares y el desarrollo de las emociones en los individuos durante la infancia. Por ejemplo, y en un sentido positivo, Lekaviciene y Antiniene (2016) señalan que la familia cumple un rol importante en el desarrollo de la personalidad; mientras que, según Gottman (2001), los padres son los entrenadores de las emociones de los niños y contribuyen en el entendimiento y reconocimiento de los efectos negativos, el desarrollo del sentido de control y optimismo del niño, y en la regulación eficaz de sus emociones.

Asimismo, pero en sentido negativo, algunos autores creen que los conflictos familiares promueven el descontrol emocional de los niños (Davies \& Cummings, 1994), que los conflictos maritales y la disfunción familiar pueden ser predictores de los desajustes emociones de los hijos (Jadue, 2003), y que las situaciones adversas en el interior de la familia pueden predecir los problemas emocionales y de comportamiento de los niños (Bradley \& Corwyn, 2002); $\mathrm{y}$, en este sentido, los problemas familiares se constituyen como un factor de riesgo para quienes interactúan en el interior de la familia y, especialmente, para los niños. No obstante, se debe aclarar que, si bien existen niños expuestos a este factor de riesgo, también existen algunos que escapan de sus efectos (Flouri, Midouhas \& Joshi, 2015).

\section{Estructura familiar y emociones}

Por otra parte, la perspectiva de los procesos de cambio social -en especial, los de industrialización y urbanizaciónincluyen las trasformaciones de la institución familiar, en donde se han visto cambios en sus funciones y se ha abierto la posibilidad de estudiar la familia desde la perspectiva de las estructuras familiares (Paredes, 2003). Con esto, debido a que grandes proporciones de la población tenían dificultades para adaptarse a las formas de comportamiento esperadas, el modelo tradicional de familia nuclear fue perdiendo fuerza (Furstenberg, 2003), lo que llevó a que conceptos como casamiento, divorcio y monoparentalidad adquirieran nuevos significados, con contenidos distintos (Paredes, 2003). Tal es el caso de las familias monoparentales, pues, si bien la familia constituida por ambos padres sigue siendo el modelo de familia más frecuente en América Latina, también se observa un incremento de las familias que cuentan con la presencia de solo uno de ellos (Olhaberry \& Farkas, 2012).

Actualmente, la sociedad asume que la interacción de los miembros de la familia y la presencia de los padres está relacionada con el desarrollo de las emociones de los niños; por ejemplo, hay quienes sostienen que los jóvenes que conviven con ambos padres presentan una mayor satisfacción con la vida y mayor autoestima (Montoya \& Landero, 2013); así como hay autores que consideran que la presencia de la autoridad paterna en la familia, además de hacer que los niños mantengan los elementos básicos para el crecimiento social y emocional, proporciona oportunidades para que los niños se desarrollen fuera de la familia (Shapiro \& Tiscornia, 1997); y quienes afirman que la paternidad, a través de la percepción de calor -especialmente de la madre- está asociada positivamente con la inteligencia emocional, constituyéndose como uno de los factores más eficaces en el desarrollo de este tipo de inteligencia (Asghari \& Besharat, 2011). Asimismo, también se han encontrado autores que consideran que los niños se ven afectados negativamente por el divorcio de sus padres (Amato \& Keith, 1991), y que los hijos que viven con un solo padre son más proclives a experimentar ansiedad o presentar síntomas de tensión emocional (Jadue, 2003).

Teniendo todo lo anterior en cuenta, resulta evidente que la inteligencia emocional es considerada como una variable que puede ejercer alguna influencia sobre otros factores; sin embargo, son escasas las investigaciones que analizan la influencia que ejercen otras variables sobre la inteligencia emocional, y es por esta razón que en la presente investigación se propone el análisis de la inteligencia emocional y su relación con el género y la estructura y el clima familiar. 


\section{MÉTODO}

\section{Diseño}

Se llevó a cabo un estudio de enfoque cuantitativo con diseño descriptivo-correlacional.

\section{Participantes}

A partir de un muestreo intencional, participaron voluntariamente 127 adolescentes pertenecientes a una asociación educativa del distrito San Juan de Lurigancho, en Lima, Perú. En detalle, la edad promedio de los participantes fue de 14.2 años ( \pm 1.49 ), el $52 \%$ eran mujeres, y el $73.2 \%$ provenía de familias nucleares y el $26.8 \%$ de familias monoparentales (véase Tabla 1).

Tabla 1

Características sociodemográficas de los participantes

\begin{tabular}{llcc}
\hline \multicolumn{2}{c}{ Variables } & $\mathrm{n}$ & $\%$ \\
\hline Edad & $<13$ años & 51 & 40.2 \\
& $13-15$ años & 53 & 41.7 \\
\multirow{4}{*}{ Género } & $>15$ años & 23 & 18.1 \\
\multirow{4}{*}{ Grado de escolaridad } & Femenino & 66 & 52.0 \\
& Masculino & 61 & 48.0 \\
& $1^{\circ}$ secundaria & 28 & 22.0 \\
& $2^{\circ}$ secundaria & 26 & 20.5 \\
& $3^{\circ}$ secundaria & 24 & 18.9 \\
& $4^{\circ}$ secundaria & 29 & 22.8 \\
Estructura familiar & $5^{\circ}$ secundaria & 20 & 15.7 \\
& Nuclear & 93 & 73.2 \\
& Monoparental & 34 & 26.8 \\
\hline
\end{tabular}

Nota . Total $=127$ adolescentes.

\section{Instrumentos}

Para la medición del clima familiar se hizo una reducción y adaptación de la escala de Clima Social Familiar de Moos y Tricket (1987), instrumento validado que originalmente consta de 90 ítems con opciones de respuestas dicotómicas (verdadero-falso) y que mide los componentes de: relaciones -que se refiere al grado de comunicación, libertad y posibilidad de expresión en el hogar-, desarrollo -que denota la realización personal de cada miembro de la familia-, y estabilidad -que evalúa la organización y respeto por las reglas establecidas el interior de la familia-.

Específicamente, la escala se redujo a 60 ítems con opciones de respuesta tipo Likert ( $1=$ nunca, $2=$ a veces, $3=$ casi siempre, $4=$ siempre), lo que permitió realizar un análisis de fiabilidad a través del coeficiente alfa (Cronbach, 1951), además de, a partir de las recomendaciones de DomínguezLara (2016), un cálculo de los intervalos de confianza (IC) que permitió corroborar una adecuada consistencia interna entre el total de sus ítems $\left(\alpha=.898\right.$; $\left.\mathrm{IC}_{(95 \%)}=.858-.927\right)$; y el total de las dimensiones: relaciones $\left(\alpha=.815\right.$; $\mathrm{IC}_{(95 \%)}$ $=.747-.866)$, desarrollo $\left(\alpha=.800, \mathrm{IC}_{(95 \%)}=.727-.855\right)$ y estabilidad $\left(\alpha=.788, \mathrm{IC}_{(95 \%)}=.711-.846\right)$. Resultados similares a los reportados en otros estudios (AlDosiry et al., 2015; Extremera \& Fernández, 2004).

Por otra parte, la inteligencia emocional fue evaluada a través de una escala propuesta y validada por Chiriboga y Franco (2001), que consta de 60 ítems con opciones de respuesta de tipo Likert ( $1=$ nunca, $2=$ a veces, $3=$ casi siempre, $4=$ siempre) para evaluar los cinco componentes propuestos por Goleman (2012): autoconciencia, que mide la habilidad para conocerse uno mismo, en especial las emociones y la interpretación adecuada de la realidad que lo rodea; autorregulación, la actuación de forma coherente con las convicciones y experiencias personales, además de saber adaptarse a los diferentes contextos, innovando pero sin perder la integridad personal; automotivación, la capacidad para motivarse, definir objetivos, metas y el compromiso con el logro de las mismas, siendo conscientes del tiempo y el esfuerzo que puede llevar alcanzarlas; empatía, la capacidad para reconocer las emociones de los demás y adaptarse a diferentes personas sin perder la identidad personal ni el control emocional; y habilidades sociales, el control de las relaciones sociales como un conjunto de habilidades que facilitan y permiten la adaptación al grupo, la colaboración en equipos, la resolución de conflictos y la comunicación eficiente.

En la muestra estudiada, el análisis de fiabilidad para la escala total de la inteligencia emocional arrojó un coeficiente de fiabilidad que muestra una adecuada consistencia interna entre sus elementos $\left(\alpha=.860, \mathrm{IC}_{(95 \%)}=.807-.899\right)$.

\section{Procedimiento}

Para la recolección de los datos se solicitó la autorización a la directiva institucional y a los docentes de cada aula de clases. Una vez autorizada la investigación, se le entregó un ejemplar de los instrumentos impresos y un lapicero a cada adolescente en su salón de clases, y se dieron las orientaciones sobre el contenido, propósito y modo de respuestas a los ítems de los mismos. El tiempo estimado para responder fue de 25 minutos.

\section{Consideraciones éticas}

Se solicitó la firma del consentimiento informado por parte de los padres de familia de los participantes con el objetivo de que los adolescentes y sus progenitores conocieran los fines del estudio y aceptaran participar voluntariamente bajo las condiciones de respetar su privacidad y la confidencialidad de la información, así como 
el compromiso de presentar los resultados individuales y colectivos para que la directiva institucional pudiera tomar decisiones o realizar acciones, basadas en evidencias, que favorecieran el bienestar emocional y familiar de los adolescentes. Los adolescentes que manifestaron sentirse inseguros o temerosos de brindar información acerca de su familia no participaron del estudio; esto evitó posibles efectos emocionales negativos a causa del recuerdo.

\section{Análisis estadístico}

Antes de realizar el análisis estadístico para las diferencias de género y estructura familiar en cuanto a la inteligencia emocional y sus respectivos factores, se realizó un análisis de normalidad a través de la prueba de Shapiro-Wilk. Si bien se considera que la sensibilidad de todas las pruebas estadísticas de normalidad depende del tamaño de la muestra, la evidencia obtenida por estudios de simulaciones muestra que la prueba Shapiro-Wilk tiene un mejor rendimiento en la mayoría de las situaciones (Yap \& Sim, 2011).

Los resultados del análisis de normalidad indican que la inteligencia emocional y sus respectivos componentes en las categorías del género presentan una distribución normal $(p>.05)$, al igual que en las categorías de la estructura familiar ( $p>.05)$, pero no en las categorías de estructura familiar de tipo nuclear y monoparental, donde los datos para autorregulación y habilidades sociales, respectivamente, no se distribuyeron de manera normal $(\mathrm{p}<.05)$ (véase Tabla 2$)$.

Por este motivo, se decidió realizar el análisis estadístico a partir de la prueba $t$ de Student para analizar la diferencia de medias en los componentes de la inteligencia emocional entre adolescentes mujeres y hombres, y entre adolescentes que proceden de familias nucleares y monoparentales.
Además, se consideró realizar el cálculo de la potencia de prueba - para evitar cometer un error de tipo I-, utilizando el estadístico d de Cohen (1992), una medida de la magnitud del efecto pertinente para un procedimiento comparativo (Dominguez-Lara, 2017). La valoración en esta medida oscila entre: insignificante $(<.20)$, pequeña $(.20)$, mediana (.50) y grande (.80).

El análisis de correlaciones entre los componentes de la inteligencia emocional y el clima familiar se hizo a través de la prueba estadística $r$ de Pearson, donde se estableció un nivel de significancia de .05 (5 \%). A su vez, la r fue valorada como magnitud del efecto en sí misma (Cohen, 1988), según los criterios: $<.10=$ insignificante, $.10=$ pequeña, $.30=$ mediana y $.50=$ grande. Complementariamente, se compararon los $\mathrm{r}$ entre varones y mujeres por medio del estadístico q (Cohen, 1992; Dominguez, Moscoso, Merino \& Navarro, 2016), y se valoró la magnitud del efecto con los criterios previamente utilizados. Los análisis estadísticos se realizaron por medio del software SPSS 22.0.

\section{RESULTADOS}

Acontinuación, se presentan los resultados de la comparación de medias según el género y el coeficiente de Cohen, seguidos de los análisis de correlación entre inteligencia emocional y clima familiar.

Específicamente, el análisis de contraste de medias confirmó la existencia de diferencias significativas en las puntuaciones de empatía $(\mathrm{t}=3.445 ; \mathrm{p}<.01)$ y habilidades sociales $(t=2.711 ; p<.01)$ de las mujeres, en comparación con los hombres. Estos resultados muestran que las

Tabla 2

Análisis de normalidad de la inteligencia emocional según género y estructura familiar

\begin{tabular}{|c|c|c|c|c|c|c|c|c|}
\hline \multirow{2}{*}{ Variables } & \multicolumn{4}{|c|}{ Género } & \multicolumn{4}{|c|}{ Estructura familiar } \\
\hline & Categoría & $\mathrm{W}$ & $\mathrm{gl}$ & Sig. & Categoría & $\mathrm{W}$ & $\mathrm{gl}$ & Sig \\
\hline \multirow[t]{2}{*}{ Autoconocimiento } & Femenino & .986 & 66 & .673 & Nuclear & .980 & 93 & .167 \\
\hline & Masculino & .977 & 61 & .306 & Monoparental & .962 & 34 & .283 \\
\hline \multirow[t]{2}{*}{ Autorregulación } & Femenino & .964 & 66 & .055 & Nuclear & .969 & 93 & .028 \\
\hline & Masculino & .981 & 61 & .448 & Monoparental & .941 & 34 & .064 \\
\hline \multirow[t]{2}{*}{ Automotivación } & Femenino & .967 & 66 & .075 & Nuclear & .974 & 93 & .065 \\
\hline & Masculino & .975 & 61 & .238 & Monoparental & .957 & 34 & .197 \\
\hline \multirow[t]{2}{*}{ Empatía } & Femenino & .984 & 66 & .540 & Nuclear & .986 & 93 & .399 \\
\hline & Masculino & .970 & 61 & .144 & Monoparental & .947 & 34 & .097 \\
\hline \multirow[t]{2}{*}{ Habilidades sociales } & Femenino & .970 & 66 & .108 & Nuclear & .986 & 93 & .443 \\
\hline & Masculino & .994 & 61 & .993 & Monoparental & .925 & 34 & .022 \\
\hline \multirow[t]{2}{*}{ Inteligencia emocional } & Femenino & .977 & 66 & .262 & Nuclear & .991 & 93 & .816 \\
\hline & Masculino & .984 & 61 & .591 & Monoparental & .971 & 34 & .503 \\
\hline
\end{tabular}

Nota. $\mathrm{W}=$ Test de Shapiro-Wilk. 
mujeres presentan niveles de empatía y de habilidades sociales más altos que sus pares hombres $(d=.62$ y $d=$ .49 , respectivamente), pero no se encontraron diferencias estadísticamente significativas $(\mathrm{p}>.05)$ en la puntuación total de la inteligencia emocional, ni en los componentes interpersonales de autoconocimiento, autorregulación y automotivación; aunque la magnitud del efecto para la variable de autoconocimiento no es despreciable $(d=.34)$ y para la inteligencia emocional se encuentra dentro del límite $(d=.20)$ (véase Tabla 3$)$.

Con respecto al contraste de medias de la inteligencia emocional entre adolescentes que provienen de familias nucleares o monoparentales, se encontraron diferencias significativas entre las puntuaciones de autorregulación $(\mathrm{t}=2.319 ; p<.05)$ y automotivación $(\mathrm{t}=2.713 ; p<.01)$, es decir, que los provenientes de familias nucleares presentaron puntuaciones de autorregulación $(\bar{x}=2.78 ; \pm .31)$ superiores a las de quienes provienen de familias monoparentales $(\bar{x}=2.63 ; \pm .32)$. Asimismo, las puntuaciones de automotivación para los adolescentes de familias nucleares $(\bar{x}=3.13 ; \pm .41)$ fueron superiores en comparación con los de familias monoparentales $(\bar{x}=2.90 ; \pm .46)$. Sin embargo, no se observaron diferencias significativas $(\mathrm{p}>.05)$ en la inteligencia emocional general, ni en los componentes de autoconocimiento, empatía y habilidades sociales (véase Tabla 4).

Por otra parte, en la Tabla 5 se muestra el análisis estadístico a través del coeficiente $r$ de Pearson, el cual muestra una correlación directa y estadísticamente significativa entre el clima familiar y la inteligencia emocional $(\mathrm{r}=.632 ; \mathrm{p}<.01) ; \mathrm{y}$, específicamente, en los análisis de relaciones entre los componentes del clima familiar y la inteligencia emocional se encontraron relaciones directas y estadísticamente significativas $(\mathrm{p}<.01)$, aunque con la valoración de los $\mathrm{r}$, desde el enfoque de magnitud del efecto, todas las correlaciones presentaron magnitudes que permiten la interpretación teórica de los resultados (>.20).

En cuanto a la comparación de correlaciones de la inteligencia emocional y el clima familiar entre hombres y mujeres, se puede observar que no hay diferencias en la mayoría de las correlaciones analizadas, lo que indica que el clima familiar tiene una relación similar con la inteligencia emocional, independientemente del género de la persona (véase Tabla 6). Sin embargo, se pueden tener en cuenta

Tabla 3

Inteligencia emocional entre adolescentes mujeres y hombres

\begin{tabular}{llllllllccc}
\hline & $\begin{array}{l}\text { Mujeres } \\
(\mathrm{n}=66)\end{array}$ & \multicolumn{3}{c}{$\begin{array}{c}\text { Hombres } \\
(\mathrm{n}=61)\end{array}$} & \multicolumn{7}{c}{ Prueba t para la diferencia de medias } \\
& Media & DE & Media & DE & Diferencia & Inferior & Superior & $\mathrm{t}$ & $\mathrm{p}$ & $\mathrm{d}$ \\
\hline Autoconocimiento & 2.80 & .37 & 2.92 & .34 & -.120 & -.247 & .006 & -1.887 & .062 & .34 \\
Autorregulación & 2.74 & .36 & 2.74 & .27 & .001 & -.110 & .113 & .025 & .980 & .00 \\
Automotivación & 3.06 & .43 & 3.07 & .44 & -.007 & -.160 & .145 & -.095 & .924 & .02 \\
Empatía & 3.20 & .34 & 2.97 & .40 & .229 & .097 & .360 & 3.445 & .001 & .62 \\
Habilidades sociales & 3.24 & .44 & 3.03 & .41 & .205 & .055 & .354 & 2.711 & .008 & .49 \\
IE & 3.01 & .30 & 2.95 & .29 & .061 & -.043 & .165 & 1.167 & .245 & .20 \\
\hline
\end{tabular}

Nota. DE: Desviación estándar; $d=d$ de Cohen.

Tabla 4

Inteligencia emocional entre adolescentes de familias nucleares y monoparentales

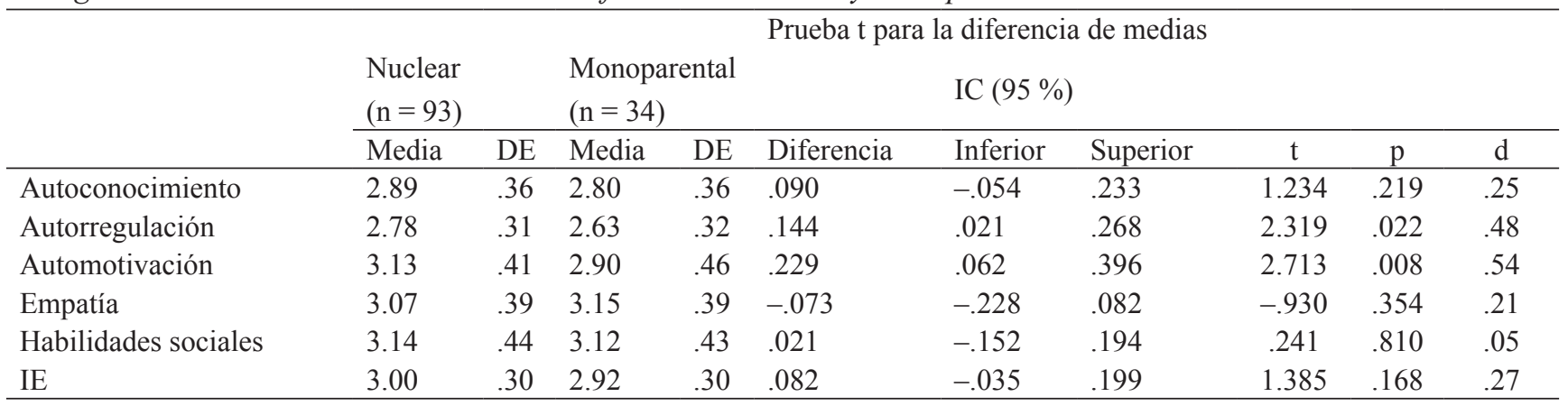

Nota. DE: Desviación estándar; $d: d$ de Cohen. 
Tabla 5

Correlaciones entre los componentes del CF y la IE

\begin{tabular}{lcccc}
\hline & Relación & Desarrollo & Estabilidad & Clima familiar \\
\hline Autoconocimiento & $.602^{* *}$ & $.450^{* *}$ & $.396^{* *}$ & $.576^{* *}$ \\
Autorregulación & $.453^{* *}$ & $.360^{* *}$ & $.263^{* *}$ & $.435^{* *}$ \\
Automotivación & $.603^{* *}$ & $.465^{* *}$ & $.440^{* *}$ & $.597^{* *}$ \\
Empatía & $.343^{* *}$ & $.379^{* *}$ & $.310^{* *}$ & $.416^{* *}$ \\
Habilidades sociales & $.347^{* *}$ & $.356^{* *}$ & $.248^{* *}$ & $.388^{* *}$ \\
Inteligencia emocional & $.612^{* *}$ & $.527^{* *}$ & $.436^{* *}$ & $.632^{* *}$ \\
\hline
\end{tabular}

Nota. $*: \mathrm{p}<.05 ; * * \mathrm{p}<.01$.

Tabla 6

Comparación de correlaciones de CF y la IE entre hombres y mujeres

\begin{tabular}{|c|c|c|c|c|c|}
\hline & & Relación & Desarrollo & Estabilidad & Clima familiar \\
\hline \multirow[t]{3}{*}{ Autoconocimiento } & Hombres & $.500 * *$ & $.305^{*}$ & $.284 *$ & $.444 * *$ \\
\hline & Mujeres & $.681 * *$ & $.562 * *$ & $.457 * *$ & $.672 * *$ \\
\hline & $\mathrm{q}$ & .130 & .214 & .151 & .169 \\
\hline \multirow[t]{3}{*}{ Autorregulación } & Hombres & $.286^{*}$ & $.397 * *$ & .212 & $.385 * *$ \\
\hline & Mujeres & $.553 * *$ & $.340 * *$ & $.290 *$ & $.462 * *$ \\
\hline & $q$ & .224 & .050 & .073 & .065 \\
\hline \multirow[t]{3}{*}{ Automotivación } & Hombres & $.548 * *$ & $.360 * *$ & $.420 * *$ & $.529 * *$ \\
\hline & Mujeres & $.652 * *$ & $.548^{* *}$ & $.464 * *$ & $.655^{* *}$ \\
\hline & $\mathrm{q}$ & .074 & .154 & .036 & .090 \\
\hline \multirow[t]{3}{*}{ Empatía } & Hombres & $.271 *$ & $.381 * *$ & $.298 *$ & $.392 * *$ \\
\hline & Mujeres & $.469 * *$ & $.415^{* *}$ & $.407 * *$ & $.503 * *$ \\
\hline & $\mathrm{q}$ & .173 & .030 & .096 & .091 \\
\hline \multirow[t]{3}{*}{ Habilidades sociales } & Hombres & $.329 * *$ & $.380 * *$ & $.477 * *$ & $.464 * *$ \\
\hline & Mujeres & $.392 * *$ & $.353 * *$ & .154 & $.371 * *$ \\
\hline & $\mathrm{q}$ & .056 & .024 & .291 & .078 \\
\hline \multirow[t]{3}{*}{ Inteligencia emocional } & Hombres & $.511 * *$ & $.473 * *$ & $.456^{* *}$ & $.583 * *$ \\
\hline & Mujeres & $.701 * *$ & $.569 * *$ & $.449 * *$ & $.680 * *$ \\
\hline & $\mathrm{q}$ & .134 & .074 & .006 & .067 \\
\hline
\end{tabular}

Nota. ${ }^{*}: \mathrm{p}<.05 ; *: \mathrm{p}<.01$.

tres diferencias relevantes $(q>.20)$ en las correlaciones entre autoconocimiento y desarrollo, entre autorregulación y relación, y entre habilidades sociales y estabilidad. El estadístico que evalúa la magnitud del efecto indica que en un grupo las correlaciones entre las variables analizadas son más fuertes que el otro.

\section{DISCUSIÓN}

El propósito de este estudio fue analizar la relación entre la inteligencia emocional, el género, la estructura familiar y el clima familiar en adolescentes del distrito de San Juan de Lurigancho, en Lima, Perú. Los resultados demuestran la existencia de diferencias de género en los niveles de empatía, donde las mujeres presentan niveles superiores a los hombres. Estos resultados coinciden con el estudio de González y Valdez (2013), en el que se encuentra que las mujeres tienen niveles de empatía significativamente superiores a los hombres.

Al respecto, Batson, Fultz y Schoenrade (1987) sostienen que los estereotipos sociales atribuyen a la mujer una mayor sensibilidad emocional, tendencia al cuidado y apoyo a los más débiles, mayor capacidad para detectar sentimientos y señales no verbales, y una mayor preocupación por los otros. E, incluso, hay evidencia que soporta la idea de que la empatía es una característica que se atribuye frecuentemente a las mujeres, y no tanto a los hombres (González \& Valdez, 2013).

Así mismo, los resultados de este estudio aportan evidencia sobre las diferencias en habilidades sociales según el género, ya que las mujeres presentaron puntuaciones significativamente más altas que los varones. Asimismo, estudios como los de DiPrete y Jennings (2012) o Downey 
y Vogt (2005) sostienen que, si bien las diferencias de género en las habilidades sociales atribuyen ventajas para las mujeres frente a los hombres, también la variable de la edad tiene una gran importancia, en el sentido de que las estudiantes adolescentes mujeres tienen mejores habilidades sociales en edades más avanzadas que los hombres.

Por otra parte, no se encontraron diferencias significativas entre los estudiantes con respecto al autoconocimiento de las emociones; resultados similares a los de Lahav, Maeir y Weintraub (2014), donde se manifiesta que no existen diferencias de género en relación con el autoconocimiento de los estudiantes sobre su rendimiento.

Asimismo, tampoco se observaron diferencias de género con respecto al autocontrol de las emociones; sin embargo, los estudios de McKinley et al. (2014) muestran que los hombres presentan puntuaciones más altas que las mujeres en esta variable. Las discrepancias entre estos estudios y la inexactitud de las diversas investigaciones que demuestran las diferencias de género, atribuyendo a las mujeres una mayor emotividad respecto a los hombres (Else, Higgins, Allison \& Morton, 2012), evidencian la necesidad de estudiar con mayor profundidad las diferencias de género en el control de las emociones.

De igual forma, tampoco se encontraron diferencias de género con respecto a la automotivación de los adolescentes, aunque en la revisión de literatura realizada por Meece, Glienke y Burg (2006) hay evidencia sobre la existencia de diferencias en la capacidad de concentración, en el aprendizaje de las ciencias, aunque no en cuanto a las orientaciones de meta entre estudiantes americanos europeos, que tomados en conjunto no revelan un patrón claro de las diferencias de género en la orientación de meta hacia el logro.

Adicionalmente, con respecto a la relación entre la estructura familiar y la inteligencia emocional, se observó que los adolescentes que provenían de familias nucleares presentaban puntuaciones de autocontrol y automotivación más altas que aquellos que provenían de familias monoparentales; resultados que concuerdan con lo encontrado por Pérez et al. (2009), donde se afirma sobre el autocontrol de las emociones que la estructura de la familia cumple un rol fundamental en la funcionalidad de la familia, y que la familia nuclear está mejor preparada para afrontar los cambios que ocurren entre sus miembros. Adicionalmente, según Santander et al. (2008), los adolescentes que perciben la disfuncionalidad de sus familias son más vulnerables a las conductas de riesgo, lo cual refleja la pérdida del control de emociones, ya que, según Meece et al. (2006), la familia cumple una función básica en la formación de creencias e intereses de competencia, gracias a que la socialización y las experiencias que se manifiestan en la familia influyen en las diferencias de género con respecto a aspectos motivacionales que se desarrollan a temprana edad.

Finalmente, en el análisis de correlación se encontró la existencia de una relación directa y significativa entre el clima familiar y la inteligencia emocional, ya que los estudiantes que evidenciaron puntuaciones altas en el clima familiar también lo hicieron en la inteligencia emocional. De manera similar, se observaron correlaciones altas y directas entre las dimensiones de clima familiar y las dimensiones de las variables de la inteligencia emocional.

A pesar de que no se encontraron estudios sobre la influencia del clima familiar en la inteligencia emocional, sí hay evidencia sobre la influencia de variables de la familia sobre el control de las emociones de los adolescentes, como es el caso del estudio de Ramsden y Hubbard (2002), donde se muestra que la expresividad familiar negativa y la aceptación de las emociones negativas de la madre se relacionan indirectamente con el comportamiento agresivo a través del autocontrol emocional de los niños; o el de Davies y Cummings (1994), donde se observó que el control emocional de los niños está asociado a sus experiencias relacionadas con los conflictos de pareja de sus padres.

Con respecto a las limitaciones del presente estudio, a pesar de que tanto los coeficientes de fiabilidad calculados (incluyendo el IC con límite inferior por encima de .70) como las correlaciones halladas entre las dimensiones estudiadas sugieren que el tamaño de la muestra no afectó sistemáticamente los hallazgos, el tamaño muestral utilizado $(<200)$ es modesto para un estudio correlacional, y sería necesario replicar el estudio con una muestra mayor con el fin de consolidar los resultados.

Por otra parte, un aspecto que contribuyó a la robustez de los resultados fue el uso de medidas de magnitud del efecto, las cuales permitieron conocer las asociaciones entre las variables -tanto en el análisis comparativo como en el correlacional- más allá del tamaño muestral que, como se sabe, afecta el valor $\mathrm{p}$-lo que lleva a retener o no a la hipótesis nula estadística-, pudiendo arribar a conclusiones que podrían reflejar inapropiadamente los resultados reales (Dominguez-Lara, 2016). Por tanto, se sugiere el desarrollo de futuras investigaciones que analicen el efecto moderador del género y la estructura familiar en la relación del clima familiar y la inteligencia emocional.

Asimismo, sería importante emprender estudios con diseños longitudinales que permitan analizar las diferencias de la inteligencia emocional y el clima familiar en adolescentes que proceden de un determinado grupo familiar. A pesar de las limitaciones, se considera que los resultados obtenidos aportan a un mejor conocimiento sobre la relación de las variables expuestas en este grupo de personas. 
En síntesis, los resultados ponen de manifiesto que la inteligencia emocional proporciona competencias para afrontar situaciones de estrés y relacionamiento social, y que las habilidades que permiten comprender, controlar y discernir entre diferentes estados emocionales son imprescindibles para el desarrollo social del individuo y se constituyen como factores de protección para la salud mental y física de los individuos. Cabe mencionar, por último, que es necesario seguir investigando acerca de las emociones, ya que niveles demasiado altos o bajos podrían generar dificultades en las relaciones e interacción social de todo tipo de personas (Salguero \& Iruarrizaga, 2006).

\section{REFERENCIAS}

Aimaganbetova, O., Tolegenova, A., Nurysheva, G., Syrgakbaeva, A., Mussikhina, E., Dzhumagalieva, I., \& Aimaganbetov, A. (2016). The Impact of "Self-knowledge" Subject on Social-psychological Characteristics of Pupils' Identity. Procedia. Social and Behavioral Sciences, 217, 771-778. Doi: https://doi.org/10.1016/j.sbspro.2016.02.143

AlDosiry, K. S., Alkhadher, O. H., AlAqraa', E. M., \& Anderson, N. (2015). Relationships between emotional intelligence and sales performance in Kuwait. Revista de Psicología Del Trabajo Y de Las Organizaciones, 32(1), 39-45. Doi: https://doi.org/10.1016/j.rpto.2015.09.002

Amato, P. R., \& Keith, B. (1991). Parental divorce and the wellbeing of children: a meta-analysis. Psychological Bulletin, 110(1), 26-46.

Argyle, M. (1990). The psychology of interpersonal behaviour. Harmondsworth, UK: Penguin.

Aritzeta, A., Balluerka, N., Gorostiaga, A., Alonso-Arbiol, I., Haranburu, M., \& Gartzia, L. (2016). Classroom emotional intelligence and its relationship with school performance. European Journal of Education and Psychology, 9(1), 1-8. Doi: https://doi.org/10.1016/j.ejeps.2015.11.001

Asghari, M. S., \& Besharat, M. A. (2011). The relation of perceived parenting with emotional intelligence. Procedia. Social and Behavioral Sciences, 30, 231-235. Doi: https://doi. org/10.1016/j.sbspro.2011.10.046

Azpiazu, L., Esnaola, I., \& Sarasa, M. (2015). Capacidad predictiva del apoyo social en la inteligencia emocional de adolescentes. European Journal of Education and Psychology, 8(1), 23-29. Doi: https://doi.org/10.1016/j.ejeps.2015.10.003

Bagozzi, R. P., Gopinath, M., \& Nyer, P. U. (1999). The Role of Emotions in Marketing. Journal of the Academy of Marketing Science, 27(2), 184-206. Doi: https://doi. org/10.1177/0092070399272005

Bar-On, R. (1997). EQ-I Bar-On Emotional Quotient Inventory: A Measure of Emotional Intelligence. Multi-Health Systems.
Batson, C. D., Fultz, J., \& Schoenrade, P. A. (1987). Las reacciones emocionales de los adultos ante el malestar ajeno. En N. Eisenberg \& J. Strayer (Eds.), La empatía y su desarrollo (pp. 181-204). Bilbao: Desclée de Brouwer.

Bradley, R. H., \& Corwyn, R. F. (2002). Socioeconomic status and child development. Annual Review of Psychology, 53, 371-99. Doi: https://doi.org/10.1146/annurev. psych.53.100901.135233

Chester, D. S., Lynam, D. R., Milich, R., Powell, D. K., Andersen, A. H., \& De Wall, C. N. (2016). How Do Negative Emotions impair self-control? A neural model of negative urgency. NeuroImage, 132, 43-50. Doi: https://doi.org/10.1016/j. neuroimage.2016.02.024

Chiriboga, R., \& Franco, J. (2001). Validación de un test de inteligencia emocional en niños de diez años de edad. Médico de Familia, 1-7.

Cohen, J. (1988). Statistical power analysis for the behavioral sciences (2da. ed.). New York: Lawrence Erlbaum Associates.

Cohen, J. (1992). A power primer. Psychological Bulletin, 112(1), 155-9. Recuperado de http://www.ncbi.nlm.nih. gov/pubmed/19565683

Conway, J., Bourque, S., \& Scott, J. (2013). El concepto de género. En M. Lamas (Ed.), El género: la construcción cultural de la diferencia sexual (pp. 21-33). México: Grupo Editorial Miguel Ángel Porrúa.

Cooper, R. K., \& Sawaf, A. (1998). Executive EQ: Emotional Intelligence in Leadership and Organizations. Penguin.

Cronbach, L. J. (1951). Coefficient alpha and the internal structure of tests. Psychometrika, 16(3), 297-334. Doi: https:// doi.org/10.1007/BF02310555

Davies, P. T., \& Cummings, E. M. (1994). Marital conflict and child adjustment: an emotional security hypothesis. Psychological Bulletin, 116(3), 387-411.

DiPrete, T. A., \& Jennings, J. L. (2012). Social and behavioral skills and the gender gap in early educational achievement. Social Science Research, 41(1), 1-15. Doi: https://doi. org/10.1016/j.ssresearch.2011.09.001

Dominguez-Lara, S. (2016). Diferencias entre grupos y magnitud del efecto: un análisis complementario. Archivos Argentinos de Pediatría, 114(4), e300-e301.

Dominguez-Lara, S. (2017). Magnitud del efecto, una guía rápida. Educación Médica, En prensa, 1-4. Doi: https://doi. org/10.1016/j.edumed.2017.07.002

Domínguez-Lara, S. (2016). Anales del sistema sanitario de Navarra. Anales Del Sistema Sanitario de Navarra, 39(1), 169-170. Recuperado de http://scielo.isciii.es/scielo. php?script $=$ sci_arttext\&pid=S1137-66272016000100024

Dominguez, S., Moscoso, M. S., Merino, C., \& Navarro, J. S. (2016). Un método para la comparación de correlaciones basado en intervalos de confianza: aportes a Tejedor et al. Revista de Psiquiatría Y Salud Mental, 9(4), 228-229. Doi: https://doi.org/10.1016/j.rpsm.2015.12.001 
Downey, D. B., \& Vogt, A. S. (2005). Sex Differences In School Performance During High School: Puzzling Patterns and Possible Explanations. The Sociological Quarterly, 46(2), 299-321. Doi: https://doi.org/10.1111/j.15338525.2005.00014.x

Eagly, A. H., Beall, A. E., \& Sternberg, R. J. (2004). The psychology of gender (2nd ed.). London: Guilford Press.

Egger, H. L., \& Angold, A. (2006). Common emotional and behavioral disorders in preschool children: presentation, nosology, and epidemiology. Journal of Child Psychology and Psychiatry, and Allied Disciplines, 47(3-4), 313-37. Doi: https://doi.org/10.1111/j.1469-7610.2006.01618.x

Eisenberg, N., Spinrad, T. L., \& Eggum, N. D. (2010). Emotion-related self-regulation and its relation to children's maladjustment. Annual Review of Clinical Psychology, 6, 495-525. Doi: https://doi.org/10.1146/annurev. clinpsy. 121208.131208

Else, N., Higgins, A., Allison, C., \& Morton, L. C. (2012). Gender differences in self-conscious emotional experience: a meta-analysis. Psychological Bulletin, 138(5), 947-81. Doi: https://doi.org/10.1037/a0027930

Extremera, N., \& Fernández, P. (2004). El papel de la inteligencia emocional en el alumnado: evidencias empíricas. Revista Electrónica de Investigación Educativa. Recuperado de http://redie.uabc.mx/redie/article/view/105/1121

Fernández, P., \& Extremera, N. (2013). La Inteligencia emocional como una habilidad esencial en la escuela. Revista Iberoamericana de educación, 29(1), 1-6.

Flouri, E., Midouhas, E., \& Joshi, H. (2015). Family and neighbourhood risk and children's problem behaviour: The moderating role of intelligence. Intelligence, 53, 33-42. Doi: https://doi.org/10.1016/j.intell.2015.08.003

Furstenberg, F. (2003). El cambio familiar estadounidense en el último tercio del siglo XX. En Nuevas formas de familia: perspectivas nacionales e internacionales (pp. 11-35). Montevideo: Fondo de las Naciones Unidas para la Infancia (UNICEF).

Gardner, H. (2006). Multiple Intelligences: New Horizons. New York: Basic Books.

Goleman, D. (2011). Working With Emotional Intelligence. New York: Bantam Books.

Goleman, D. (2012). Inteligencia emocional. Barcelona: Editorial Kairós.

González, N., \& Valdez, J. (2013). Resiliencia: Diferencias por edad en hombres y mujeres mexicanos. Acta de Investigación Psicológica, 3(1), 941-955.

Gottman, J. (1997). The Heart of Parenting: How to Raise an Emotionally Intelligent Child. New York: Bloomsbury.

Gottman, J. (2001). Meta-Emotion, Children's Emotional Intelligence, and Buffering Children from Marital Conflict. En C. D. Ryff \& B. Singer (Eds.), Emotion, social relationships, and health. Series in affective science (pp. 23-55).
New York: Oxford University Press. Doi: https://doi. org/10.1093/acprof:oso/9780195145410.001.0001

Herpertz, S., Schütz, A., \& Nezlek, J. (2016). Enhancing emotion perception, a fundamental component of emotional intelligence: Using multiple-group SEM to evaluate a training program. Personality and Individual Differences, 95, 11-19. Doi: https://doi.org/10.1016/j.paid.2016.02.015

Jadue, G. (2003). Transformaciones familiares en Chile: Riesgo creciente para el desarrollo emocional, psicosocial y la educación de los hijos. Estudios Pedagógicos, 29, 115-126. Doi: https://doi.org/10.4067/S0718-07052003000100008

Johnson, A. R., \& Stewart, D. W. (2005). A Reappraisal of the Role of Emotion in Consumer Behavior. In Review of Marketing Research (Vol. 1, pp. 3-34). Emerald Group Publishing Limited. Doi: https://doi.org/10.1108/S15486435(2004)0000001005

Lafferty, J. (2004). The relationship between gender, empathy, and aggressive behaviors among early adolescents. Dissertation Abstracts International: Section B: The Sciences and Engineering, 64(12), 6377B.

Lahav, O., Maeir, A., \& Weintraub, N. (2014). Gender differences in students' self-awareness of their handwriting performance. British Journal of Occupational Therapy, 77(12), 614-618. Doi: https://doi.org/10.4276/03080221 4X14176260335309

Lekaviciene, R., \& Antiniene, D. (2016). High Emotional Intelligence: Family Psychosocial Factors. Procedia. Social and Behavioral Sciences, 217, 609-617. Doi: https://doi. org/10.1016/j.sbspro.2016.02.066

Macías, A. J., Gutiérrez, C., Carmona, F. J., \& Crespillo, D. (2015). Relationship between perceived emotional intelligence and professional quality of life with the achievement of occupational objectives in the costa del sol primary health care district. Atencion Primaria, 48(5), 301-307. Doi: https://doi.org/10.1016/j.aprim.2015.06.007

Mayer, J., Caruso, D., \& Salovey, P. (1999). Emotional intelligence meets traditional standards for an intelligence. Intelligence, 27(4), 267-298. Doi: https://doi.org/10.1016/ S0160-2896(99)00016-1

Mayer, J. D., \& Salovey, P. (1997). What is emotional intelligence? In Emotional Development and Emotional Intelligence: Educational Implications (pp. 3-31). New York: Basic Books.

McKinley, S. K., Petrusa, E. R., Fiedeldey, C., Mullen, J. T., Smink, D. S., Scott, S. E., ... Phitayakorn, R. (2014). Are There Gender Differences in the Emotional Intelligence of Resident Physicians? Journal of Surgical Education, 71(6), e33-e40. Doi: https://doi.org/10.1016/j.jsurg.2014.05.003

McNulty, J. P., Mackay, S. J., Lewis, S. J., Lane, S., \& White, P. (2015). An international study of emotional intelligence in first year radiography students: The relationship to age, gender and culture. Radiography, 22(2), 6-10. Doi: https:// doi.org/10.1016/j.radi.2015.10.008 
Med, M. (1935). Sex and Temperament in the primitive societies. New York: Morrow.

Meece, J. L., Glienke, B. B., \& Burg, S. (2006). Gender and motivation. Journal of School Psychology, 44(5), 351-373. Doi: https://doi.org/10.1016/j.jsp.2006.04.004

Mitsopoulou, E., \& Giovazolias, T. (2015). Personality traits, empathy and bullying behavior: A meta-analytic approach. Aggression and Violent Behavior, 21, 61-72. Doi: https:// doi.org/10.1016/j.avb.2015.01.007

Montoya, B. I., \& Landero, R. (2013). Satisfacción con la vida $\mathrm{y}$ autoestima en jóvenes de familias monoparentales y biparentales. Psicología Y Salud, 18(1), 117-122. Recuperado de http://revistas.uv.mx/index.php/psicysalud/article/ view/682/1202

Moos, R., \& Tricket, E. (1987). Escalas del clima social: familia, trabajo, instituciones penitenciarias, centro escolar. Madrid: TEA.

Mortan, R. A., Ripoll, P., Carvalho, C., \& Bernal, M. C. (2014). Effects of emotional intelligence on entrepreneurial intention and self-efficacy. Revista de Psicología Del Trabajo $Y$ de Las Organizaciones, 30(3), 97-104. Doi: https://doi. org/10.1016/j.rpto.2014.11.004

Olhaberry, M., \& Farkas, C. (2012). Estrés materno y configuración familiar: estudio comparativo en familias chilenas monoparentales y nucleares de bajos ingresos. Universitas Psychologicahologica, 11(4), 1317-1326.

Ordóñez, A., Maganto, C., \& González, R. (2015). Somatic complaints, emotional awareness and maladjustment in schoolchildren. Anales de Pediatría, 82(5), 308-15. Doi: https://doi.org/10.1016/j.anpedi.2014.03.020

Paredes, M. (2003). Los cambios en la familia en Uruguay: ¿Hacia una segunda transición demográfica? EnNuevas formas de familia: perspectivas nacionales e internacionales (pp. 73-101). Montevideo: Fondo de las Naciones Unidas para la Infancia (UNICEF).

Parsons, T., \& Bales, R. F. (1956). Family, socialization and interaction process. London: Routledge.

Pérez, A., Martínez, M. L., Mesa, I., Pérez, R., Leal, F. J., \& Jiménez, I. (2009). Cambios en la estructura y en la función familiar del adolescente en la última década (19972007). Atención Primaria, 41(9), 479-485. Doi: https://doi. org/10.1016/j.aprim.2009.03.015

Povedano, A., Hendry, L. B., Ramos, M. J., \& Varela, R. (2011). Victimización escolar: Clima familiar, Autoestima y Satisfacción con la vida desde una perspectiva de género. Psychosocial Intervention, 20(1), 5-12. Doi: https://doi. org/10.5093/in2011v20n1a1
Ramsden, S. R., \& Hubbard, J. A. (2002). Family Expressiveness and Parental Emotion Coaching: Their Role in Children's Emotion Regulation and Aggression. Journal of Abnormal Child Psychology, 30(6), 657-667. Doi: https://doi. org/10.1023/A:1020819915881

Riglin, L., Collishaw, S., Shelton, K. H., McManus, I. C., NgKnight, T., Sellers, R., ... Rice, F. (2016). Higher cognitive ability buffers stress-related depressive symptoms in adolescent girls. Development and Psychopathology, 28(1), 97-109. Doi: https://doi.org/10.1017/S0954579415000310

Salguero, J. M., \& Iruarrizaga, I. (2006). Relaciones entre Inteligencia Emocional percibida y emocionalidad negativa: ansiedad, ira y tristeza/depresión. Ansiedad Y Estrés, 12(2), 207-221.

Sánchez, D., León, S., \& Barragán, C. (2015). Correlación de inteligencia emocional con bienestar psicológico y rendimiento académico en alumnos de licenciatura. Investigación En Educación Médica, 4(15), 126-132. Doi: https:// doi.org/10.1016/j.riem.2015.04.002

Sánchez, M., Fernández, P., Montañés, J., \& Latorre, J. (2008). ¿Es la inteligencia emocional una cuestión de género? Socialización de las competencias emocionales en hombres y mujeres y sus implicaciones. Electronic Journal of Research in Educational Psychology, 6(15), 455-474.

Santander, S., Zubarew, T., Santelices, L., Argollo, P., Cerda, J., \& Bórquez, M. (2008). Influencia de la familia como factor protector de conductas de riesgo en escolares chilenos. Revista Médica de Chile, 136(3), 317-324. Doi: https://doi. org/10.4067/S0034-98872008000300006

Shapiro, L. E. (2010). How to Raise a Child with a High EQ: Parents' Guide to Emotional Intelligence. New York: HarperCollins.

Shapiro, L. E., \& Tiscornia, A. (1997). La inteligencia emocional de los niños. México: Vergara.

Valdivia, C. (2008). Aparecen hoy un gran número de modelos que alteran los parámetros con los que se entendía la vida familiar. Los cambios afectan a todo el sistema familiar. Revista La Revue Du REDIF, 2(1), 15-22.

Yap, B. W., \& Sim, C. H. (2011). Comparisons of various types of normality tests. Journal of Statistical Computation and Simulation, 81(12), 2141-2155. Doi: https://doi.org/10.108 $0 / 00949655.2010 .520163$ 